\title{
(3) \\ . \\ Identification and Classification of Similar Looking Food Grains
}

\author{
${ }^{1}$ P. K. Mallick, ${ }^{2}$ B.P. Mohanty \\ ${ }^{1}$ Department Computer Science and Engineering, Balasore College of Engineering and Technology, Balasore \\ ${ }^{2}$ Department of I \& CT, Fakir Mohan University, Balasore, Odisha.
}

\begin{abstract}
This paper describes the comparative study of Artificial Neural Network (ANN) and Support Vector Machine (SVM) classifiers by taking a case study of identification and classification of four pairs of similar looking food grains namely, Finger Millet, Mustard, Soyabean, Pigeon Pea, Aniseed, Cumin-seeds, Split Greengram and Split Blackgram. Algorithms are developed to acquire and process color images of these grains samples. The developed algorithms are used to extract 18 colorHSV, and 42 wavelet based texture features. Back Propagation Neural Network (BPNN)-based classifier is designed using three feature sets namely color - HSV, wavelet-texture and their combined model. SVM model for color- HSV model is designed for the same set of samples. The classification accuracies ranging from $93 \%$ to $96 \%$ for color-HSV, ranging from $\mathbf{7 8 \%}$ to $94 \%$ for wavelet texture model and from $92 \%$ to $97 \%$ for combined model are obtained for ANN based models. The classification accuracy ranging from $80 \%$ to $90 \%$ is obtained for color-HSV based SVM model. Training time required for the SVM based model is substantially lesser than ANN for the same set of images.
\end{abstract}

Keywords: Grains Identification, Color-HSV, Wavelet, BPNN, SVM

\section{INTRODUCTION}

Human inspection system has been used in the agriculture industry from long back. This system has the inherent problems of high labour cost, fatigue, inconsistency, variability, and non-availability of trained workers. Machine vision is used successfully for classification of plants, recognition of leaves, bulk grains classification, gradation, diagnosis of plant diseases, cereal grains classification and dockage identification etc,. A Machine Vision System (MVS) is a non-destructive, rapid, and cost effective technique that determines the external (HSI, size, shape, texture, surface, damages and defects) and internal characteristics (maturity, ripeness, bruises, defects, moisture) of products. It has been used to accomplish sorting and grading processes and to remove unacceptable item from products.

As, identification and classification of food grains appearing similar in nature is a difficult job for the human beings, this automated recognition system can solve the problem of misrecognition or confusion. This work can have the application in Future Robot Controlled Shopping Malls, which can provide the facility of easy marketing/shopping without the aid of more labours and also more convenient for customers.

[J. Paliwal et al, 2003] have described a method for identification of Cereal Grains and dockage using ANN [2]. [B.S Anami, et al, 2005] have described a separation, classification, and gradation of touching and bulk grain samples using artificial neural network [1]. [Younes Chtioui, et. al, 1996] have discussed, the use of ANN based colour image analysis to identify four seed varieties [9]. [S K Shah et al, 2004] have discussed design and development of a hierarchical network by incorporating textural features [5]. [Zhi-Kai Huang, 2006] has described a scheme that merges color and texture information for bark image recognition by RBPNN and SVM methodologies [10]. [Saibal Dutta, et al, 2007] have suggested Artificial Neural Network (ANN) based scheme for moment based object recognition [6]. [K Johnson, et al, 1999] have used Support Vector Machines (SVMs) in the context of face authentication [3]. [Qing Song et. al, 2002] have described a robust support vector machine for pattern classification, which experiments for the bullet hole classification problem [4]. [Sabhransu Maji, et al, 2008] have discussed straightforward classification using kernelized SVMs [7]. [V. C. Patel, et al, 1998] have discussed about detection of defects in eggs by comparing the color computer vision with ANN [8]. It is found that very little or no work is done in the area of identification and recognition of similar looking grain samples. Most of the cited work is mainly on recognition or classification of bulk grain samples and dockage detection. An attempt is made to automatic identification of food grains with similar appearance. 
The paper is organized into 5 sections. Section II gives the proposed methodology. Also contains color$\mathrm{HSV}$, wavelet based textural feature extraction process. Section III describes classification of grains using neural network and support vector machine. The results and discussions are given in section IV. Section V deals with conclusion of the work carried out.

\section{PROPOSED METHODOLOGY}

The work involves processing of images of different types of grains, extracting the features of the grains and finally developing a neural network model and support vector machine to classify the different types of grain images.

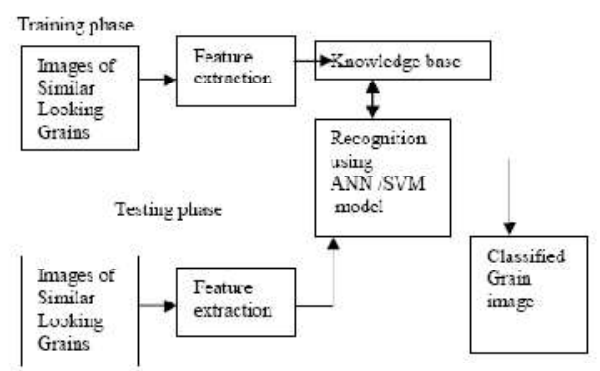

Fig. 2.1 A methodology for classification of similar looking grains.

Images of different grains are obtained using color digital camera, and the color-HSV and wavelet based texture features are extracted using image processing techniques. These features are used to train the BPNNbased classifier. SVM is trained with only color and HSV feature set. The developed classification models are thoroughly tested for different grains. The work carried out involves application of Image Processing, SVM and Neural Network techniques. The block diagram representing different phases is shown in Fig. 2.1.

\section{A. Image Acquisition}

The images are acquired by a camera connected to a personal computer (Pentium IV 2.4 GHz) mounted over the illumination chamber on a copy stand, which provided easy vertical movement to finely tune the position of the camera with respect to samples.

The different similar looking food grain samples used are Finger Millet (Eleusine coracana), Mustard(Sinapis alba), Soya Bean(Glycine max), Pigeon Peas (Cajanus cajan), Aniseed (Agastache foeniculum), Cumin seeds (Cuminum cyminum), Split Greengram (Vigna radiata) and Split Blackgram (Vigna mungo). The images of these similar looking grains are shown in Fig 2.2. A total of 1440 (180 images of each sample) images are used for the work. A set of 720 (90 images of each type) images is used as training set and another set of 720 untrained images for testing.

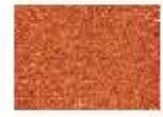

(a)

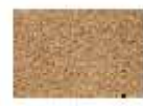

(e)

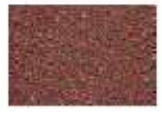

(b)

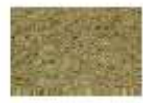

(f)

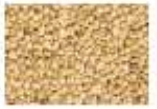

(c)

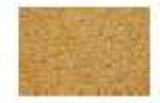

(g)

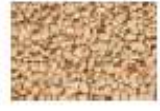

(d)

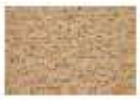

(h)

\section{B. Colour Feature Extraction}

The color features listed in Table I are obtained using Algorithm 2.1. The RGB components are separated from the image, and then Hue $(\mathrm{H})$, Saturation (S) and Value (V) components are extracted from RGB components. The equations are deployed in their evaluation of $\mathrm{H}, \mathrm{S}$ and $\mathrm{V}$ for different image samples [Rafael Gonzalez et al., 1993]. The mean, variance and range for all these 6 components are estimated and a total of 18 color features are used in training BPNN.

TABLE I : Color-HSV Features

\begin{tabular}{|c|l|c|l|}
\hline SI No. & \multicolumn{1}{|c|}{ Feature } & SI No. & \multicolumn{1}{|c|}{ Feature } \\
\hline 1 & Red mean & 10 & Hue mean \\
\hline 2 & Red variance & 11 & Hue variance \\
\hline 3 & Red range & 12 & Hue range \\
\hline 4 & Green mean & 13 & Saturation mean \\
\hline 5 & Green variance & 14 & Saturation \\
variance
\end{tabular}

\section{Algorithm 2.1: Color and HSV Feature Extraction}

Input: Original 24-bit color image.

Output: 18 color features

\section{Start}

Step 1: Separate the RGB components from the original 24-bit input color image.

Step 2: Obtain the HSV components from RGB components using the equations $2.1,2.2$ and 2.3. 


$$
\begin{gathered}
h=\left\{\begin{array}{ll}
0 & \text { if } \max =\min \\
\left(60^{\circ} \times \frac{g-b}{\max -\min }+360^{\circ}\right) \bmod 360^{\circ}, & \text { if } \max =r \\
60^{\circ} \times \frac{b-r}{\max -\min }+120^{\circ}, & \text { if } \max =g \\
60^{\circ} \times \frac{r-g}{\max -\min }+240^{\circ}, & \text { if } \max =b
\end{array} .\right. \\
s= \begin{cases}0 & \text { if } \max =\min \\
\frac{\max -\min }{\max +\min }=\frac{\max -\min }{2 l}, & \text { if } l \leq \frac{1}{2} \\
\frac{\max -\min }{2-(\max +\min )}=\frac{\max -\min }{2-2 l}, & \text { if } l>\frac{1}{2} \\
\mathrm{~V}=\max & \ldots .\end{cases}
\end{gathered}
$$

Step 3: Find the mean, variance, and range for each RGB and HSV components.

\section{Stop.}

\section{Wavelet-Textural Feature Extraction}

Some of the similar looking grain samples exhibit different textures and provide information about variations in their intensities on the surface. Multilevel Wavelet Decomposition technique is used for this purpose. After decomposition, approximate (LL) and detailed (LH, HL and $\mathrm{HH}$ ) components are obtained. After first level decomposition, the decomposition is further applied to LL component to get second level of decomposition resulting into LL1, LH1, HL1 and HH1. At third level of decomposition, LL1 is further decomposed into LL2, LH2, HL2 and HH2. Only detailed coefficients are considered for extracting features as they contain high frequency components which are rich in texture. level 1 and level 3 are considered for feature extraction. For each detailed component of level 1 and level 3, seven features are extracted, resulting into 21 features at each level and a total of 42 texture features. These features are used to train BPNN. The combination of level 1 and 3 is used, as it was found to perform well by the experiments. The list of features in Table II is extracted using Algorithm 2.2.

TABLE II : Wavelet- Texture Features

\begin{tabular}{|c|l|c|l|}
\hline $\begin{array}{c}\text { SI } \\
\text { No }\end{array}$ & \multicolumn{1}{|c|}{ Feature } & $\begin{array}{r}\text { SI } \\
\text { No }\end{array}$ & \multicolumn{1}{|c|}{ Feature } \\
\hline 1 & Vertical Mean & 12 & Horizontal MP \\
\hline 2 & $\begin{array}{l}\text { Vertical } \\
\text { Variance }\end{array}$ & 13 & $\begin{array}{l}\text { Horizontal } \\
\text { Homogeneity }\end{array}$ \\
\hline 3 & Vertical Range & 14 & Horizontal IDM \\
\hline 4 & Vertical Energy & 15 & Diagonal Mean \\
\hline 5 & Vertical MP & 16 & Diagonal Variance \\
\hline 6 & $\begin{array}{l}\text { Vertical } \\
\text { Homogeneity }\end{array}$ & 17 & Diagonal Range \\
\hline 7 & Vertical IDM & 18 & Diagonal Energy \\
\hline
\end{tabular}

\begin{tabular}{|c|l|c|l|}
\hline 8 & $\begin{array}{l}\text { Horizontal } \\
\text { Mean }\end{array}$ & 19 & Diagonal MP \\
\hline 9 & $\begin{array}{l}\text { Horizontal } \\
\text { Variance }\end{array}$ & 20 & $\begin{array}{l}\text { Diagonal } \\
\text { Homogeneity }\end{array}$ \\
\hline 10 & $\begin{array}{l}\text { Horizontal } \\
\text { Range }\end{array}$ & 21 & Diagonal IDM \\
\hline 11 & $\begin{array}{l}\text { Horizontal } \\
\text { Energy }\end{array}$ & & \\
\hline
\end{tabular}

\section{Algorithm2.2: Wavelet-based Textural Feature Extraction}

Input: Original color image.

Output: 42 features.

\section{Start}

Step 1: Convert the original color image to gray image.

Step 2: Calculate the level-1 Wavelet transform and hence decompose the signal into LL, (low frequency) and $\mathrm{LH}, \mathrm{HL}$ and $\mathrm{HH}$ (high frequency) components in horizontal, vertical and diagonal).

Step 3: Consider horizontal details only.

Step 4: Find the Mean, Variance, Range, Energy, Homogeneity, Maximum Probability, Inverse Difference Moment (IDM) are calculated by following equations.

Energy $=\sum_{x, y} P^{2}(x, y)$

Homogeneity $\left.=\sum x \sum y(P(x, y)] /(1+|x-y|)\right)$

Maximum probability $=\max (P(x, y)$

$\underset{\text { Inverse difference }}{\text { moment }}=\sum_{x, y ; x \neq y} \frac{P^{\lambda}(x, y)}{|x-y|^{k}}$

where $\mu_{x}, \mu_{y}, \sigma_{x}, \sigma_{y}$ are standard deviations defined by,

$$
\begin{aligned}
\mu_{x} & =\sum_{x} x \sum_{y} P(x, y) \\
\mu_{y} & =\sum_{y} y \sum_{x} P(x, y) \\
\sigma_{x} & =\sum_{x}\left(x-\mu_{x}\right)^{2} \sum_{y} P(x, y)
\end{aligned}
$$

Step 5: Copy the values obtained to a feature vector

Step 6: Repeat the steps 4 and step 5 for remaining detailed coefficients set namely vertical and diagonal coefficients.

Step 7: Repeat the steps 4 to 6 for third level of decomposition and combine the feature set into a single feature vector.

Stop. 


\section{GRAINS CLASSIFICATION}

This section gives the brief account of BPNN and SVM models, their training, testing and classification for similar looking grains. In this work, The Neural Network Toolbox V 4.0 for MATLAB V 7.0 is used to build the ANN models and OSU_SVM toolbox is used for the design of SVM model.

\section{A. Back Propagation Neural Network (BPNN) Model.}

The classification is carried out using three different types of feature sets, viz., color and HSV, wavelettexture and their combined model using BPNN. Databases consisting of these feature values are used to identify the grain samples. The classification of similar looking grains using SVM model for the color and HSV features is also carried out.

In color and HSV model, feature set used consists of 9 color and $9 \mathrm{HSV}$ features. A three layer BPNN is used that consists of 18 input nodes, 38 nodes in hidden layer, and 08 output nodes, for grains classification ( A feedforward 18-38-08 network). Similarly, wavelet based texture model consists of 42 features. A BPNN consists of 42 input nodes (42 texture features), 50 nodes in hidden layer and 08 outputs for grain type. A feedforward network of 42-50-08 is used for classification. The combined model feature set used consists of 9 color, $9 \mathrm{HSV}$ and 42 textural features. A feed-forward network of $60-60-08$ is used for similar grains classification.

A pattern vector of 8 bits is used to represent the output of the BPNN, where as a single bit pattern is used to represent the SVM model. The patterns for finger millet, mustard, soyabean, pigeon peas, aniseeds, cumin seeds, split green gram and split black gram for ANN are 10000000, 01000000, 00100000, 00010000, $00001000,00000100,00000010$ and 00000001 respectively. Output patterns for SVM models are 1, 2, $3,4,5,6,7$ and 8 for the same grains samples.

\section{$B$. Training and Testing for ANN and SVM Models}

In this work, 90 sample images of each grain type (totally 720 images) are considered for training the classifiers. The features extracted from these images are used to train the classifier models. After training, another set of testing images (comprising of 720 untrained images) are used to test the identification accuracy of the designed classifier models.

\section{RESULTS AND DISCUSSION}

This section gives the results of experimentation of the developed methodologies. Individually the training and testing is carried out for color, texture and combined features of using ANN. The identification accuracy of all eight different food grains using all three feature sets using ANN model are plotted as shown in Fig.4.1. Similarly, the training and testing is carried out for color and HSV based SVM model. The comparative study of both ANN and SVM color models is depicted in Fig.
4.2. The percentage of identification accuracy is defined as the ratio of correctly recognized samples to the total number of tested samples.

$$
\% \text { Accuracy of identification }=\frac{\text { Correctly recognized samples }}{\text { Number of input samples }} * 100
$$

From the results shown in Fig. 4.1 and Fig. 4.2, it is clear that the classification accuracies ranging from $93 \%$ to $96 \%$ for color-HSV, ranging from $78 \%$ to $94 \%$ for wavelet texture model and from $92 \%$ to $97 \%$ for combined model are obtained for ANN based models. Where as, classification accuracy ranging from $80 \%$ to $90 \%$ is obtained for color-HSV based SVM model.

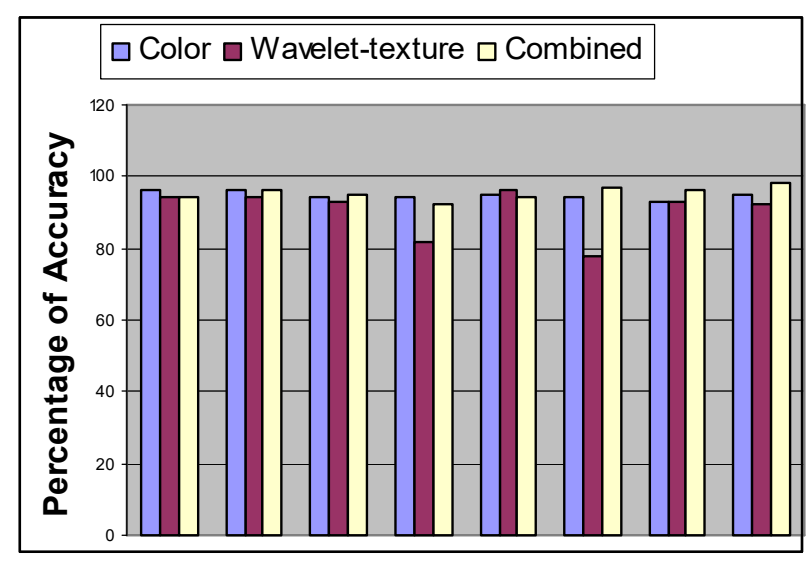

Fig 4. 1 ANN classification accuracies all the three models

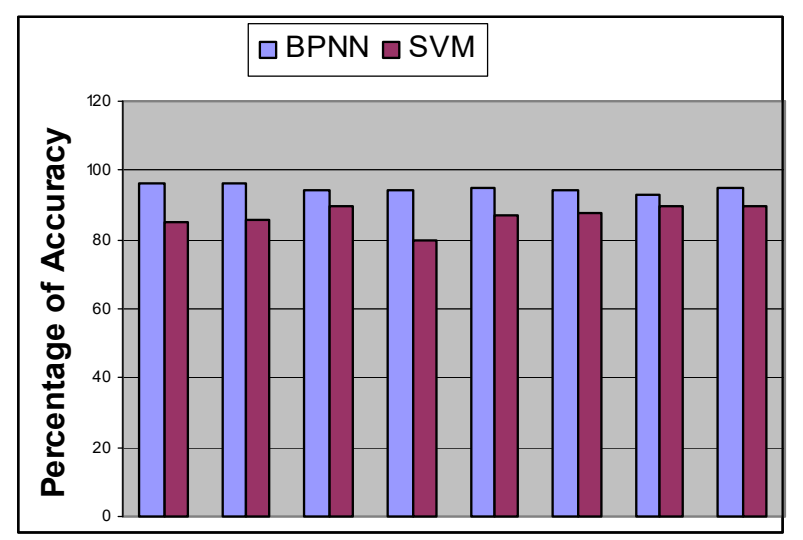

Fig 4.2 Classification accuracies of ANN and SVM using color model.

The results show that combined features perform better identification of grain type compared with individual features of ANN models. The identification accuracy will improve, when both color and textural features are considered. Hence, color and texture features are more suitable for incorporation in Machine Vision System in case of ANN model. Comparing ANN and SVM model of color features, ANN exhibits high accuracy rate than SVM. But, the time taken for training the SVM using same training set is comparatively very less. 


\section{CONCLUSIONS}

With this work, it is possible to successfully identify similar looking grains. The classification is done based on color, wavelet texture and combined features using ANN. The comparative study of color models of ANN and SVM is also carried out. The combined color and texture features are found to be more suitable for identification when ANN alone is considered. From the comparative study of ANN and SVM color models, it is observed that ANN is more accurate than SVM model. But, the time required for training SVM model is substantially less. The work carried out has relevance to real world application in identifying the similar looking food grains at robot controlled shopping malls.

\section{REFERENCES}

[1] B.S. Anami, D.G.Savakar, Aziz Makandar, and P.H. Unki (2005). A Neural Network Model for Classification of Bulk Grain Samples Based on HSI and Texture in proceedings of International Conference on Cognition and Recognition 2005, pages 359-368.

[2] J. Paliwal1, N.S. Visen1, D.S. Jayas and N.D.G. White (2003). Cereal Grain and Dockage Identification using Machine Visio. Journal of Biosystems Engineering, vol 85, pages 51- 57.

[3] K Jonsson, J Kittler, Y P Li and J Matas (1999). Support Vector Machines for Face Authentication in proceedings of British Machine Vision conference, pages 543- 553

[4] Qing Song, Wenjie Hu and Wenfang Xie (2002). Robust Support Vector Machine with Bullet hole Image Classification. IEEE Transactions on Systems, Man, and Cybernetics - Part C:
Applications and Reviews, Vol. 32, pages 440448.

[5] S K Shah and V Gandhi (2004) .Image Classification based on Textural Features Using Artificial Neural Network, Journal of Institution of Engineers (India),

[6] Saibal Dutta and P.K.Nanda (2007). Moment based Object Recognition using Artificial Neural Network, in proceedings of National conference on Intelligent Systems, pages 451- 457.

[7] Subhransu Maji, Alexander C. Berg and Jitendra Malik (2008). Classification using Intersection Kernel Support Vector Machines is Efficient. in proceedings of IEEE Computer Vision and Pattern Recognition, pages 1- 8.

[8] V. C. Patel, R.W. Mc. Clendon and J. W. Goodrum (1998), Color Computer Vision and Artificial Neural Networks for the Detection of Defects in Poultry Eggs, Journal of Artificial Intelligence Review 12, pages 163-176.

[9] Younes Chtioui, Dominique Bertrand, Yvette Datteeb and Marie-FranCoise Devaux (1996). Identification of Seeds by Color Imaging: Comparison of Discriminant Analysis and Artificial Neural Network, Journals of the science of food and agriculture, vol 71, page 433-441.

[10] Zhi-Kai Huang, Bark Classification using RBPNN based on the Color and Texture Features, International Journal of Computer Science and Network Security 2006, vol 6, page 100-103. 